OPEN ACCESS

Edited by:

Enrica Marzola,

University of Turin, Italy

Reviewed by:

Paola Longo,

University of Turin, Italy

Blake Woodside,

University Health Network

(UHN), Canada

*Correspondence:

Lisa-Katrin Kaufmann

lisa-katrin.kaufmann@usz.ch

Gabriella Franca Milos

gabriella.milos@usz.ch

Specialty section:

This article was submitted to

Psychosomatic Medicine,

a section of the journal

Frontiers in Psychiatry

Received: 19 March 2021

Accepted: 24 June 2021

Published: 15 July 2021

Citation:

Kaufmann L-K, Moergeli H and Milos GF (2021) Lifetime Weight Characteristics of Adult Inpatients

With Severe Anorexia Nervosa:

Maximal Lifetime BMI Predicts

Treatment Outcome

Front. Psychiatry 12:682952 doi: 10.3389/fpsyt.2021.682952

\section{Lifetime Weight Characteristics of Adult Inpatients With Severe Anorexia Nervosa: Maximal Lifetime BMI Predicts Treatment Outcome}

\author{
Lisa-Katrin Kaufmann ${ }^{1,2 *}$, Hanspeter Moergeli ${ }^{1}$ and Gabriella Franca Milos ${ }^{1 *}$ \\ ${ }^{1}$ Department of Consultation-Liaison Psychiatry and Psychosomatic Medicine, University Hospital Zurich, Zurich, \\ Switzerland, ${ }^{2}$ Division of Neuropsychology, Department of Psychology, University of Zurich, Zurich, Switzerland
}

Background: The body mass index is a key predictor of treatment outcome in patients with anorexia nervosa. In adolescents, higher premorbid BMI is a strong predictor of a favorable treatment outcome. It is unclear whether this relationship holds true for adults with anorexia nervosa. Here, we examine adult patients with AN and investigate the lowest and highest lifetime BMl and weight suppression as predisposing factors for treatment outcome.

Methods: We included 107 patients aged 17-56 with anorexia nervosa and tracked their BMI from admission to inpatient treatment, through discharge, to follow-up at 1-6 years. Illness history, including lowest and highest lifetime BMI were assessed prior to admission. We used multiple linear regression models with minimal or maximal lifetime $\mathrm{BMl}$ or weight suppression at admission as independent variables to predict BMl at admission, discharge and follow-up, while controlling for patients' age, sex, and duration of illness.

Results: Low minimal BMI had a negative influence on the weight at admission, which in turn resulted in a lower BMI at discharge. Higher maximal BMI had a substantial positive influence on BMl at discharge and follow-up. Weight suppression was highly correlated with maximal $\mathrm{BMI}$ and showed similar effects to maximal BMI.

Conclusion: Our findings strongly support a relationship between low minimal lifetime $\mathrm{BMl}$ and lower BMl at admission, and between higher maximal lifetime BMI or weight suppression and a positive treatment outcome, even years after discharge. Overall, maximal BMl emerged as the most important factor in predicting the weight course in adults with AN.

Keywords: anorexia nervosa, weight characteristics, hospitalization, weight suppression, treatment outcome

\section{INTRODUCTION}

Treatment for anorexia nervosa (AN) aims to restore and maintain a healthy body weight and to reduce the core psychopathology of the illness $(1,2)$, but long-term prognoses are oftentimes poor (3). The body mass index (BMI) is not only a key diagnostic measure of $\mathrm{AN}$, but also a central measure of treatment outcome. 
Premorbid BMI is assumed to be an important biological risk factor for the etiology of $\mathrm{AN}$ in adolescents, with lower premorbid BMI predicting the onset of AN (4). Previous studies in children and adolescents have suggested that higher premorbid weight acts as a protective factor for the onset of AN. For example, a large longitudinal study that tracked the BMI of children from birth to 12.5 years of age reported that the average growth trajectory of children with a subsequent onset of AN was lower than the trajectory of children who later did not develop an eating disorder (5). Premorbid BMI has been shown to be an important predictor of BMI at admission [e.g., $(6,7)]$. In adolescents, higher premorbid BMI has been shown to be predictive of a favorable treatment outcome at discharge, at 1year follow-up (6), and at 6-12-year follow-up (8). It is currently unclear if this relationship holds true for adult patients (9). In particular, it is unclear what role the longer duration of illness or the later onset of AN play with respect to the association of pretreatment weight characteristics and treatment outcome. The longer illness history of adult patients results in a more variable weight trajectory compared to adolescents. Premorbid BMI may not capture the complexity of trajectory and illness history. To account for this, the lowest and highest lifetime BMI can be used as key characteristics of past illness course.

While premorbid BMI is a measure of absolute weight status, weight suppression (the difference between highest adult weight and current or lowest weight) (10) represents a measure of relative weight status. Greater current weight suppression has been found to predict future onset of AN (11) and has been associated with faster and greater weight normalization during inpatient treatment of AN [e.g., $(12,13)]$. However, there are mixed findings regarding long-term treatment outcomes, with reports of higher weight suppression at the time of lowest BMI being associated with higher BMI at 6- to 18-year follow-up (14), and higher weight suppression at discharge predicting better weight maintenance at 1-year follow-up (15), but also reports showing no effect of weight suppression at discharge on weight maintenance at 1-year follow-up (16).

Here, we examine BMI trajectories in adult patients with AN and investigate the lowest and highest lifetime BMI, and the weight suppression at the time of lowest BMI as predisposing factors for treatment outcome. Specifically, we examine the influence of minimal lifetime BMI, maximal lifetime BMI, and maximal weight suppression on the BMI at admission to inpatient treatment, at discharge, and at 1-6-year follow-up. Patients' age, sex, and duration of illness are considered as additional predictors.

\section{METHODS}

\section{Participants and Procedure}

From January 2014 to December 2020, a total of 239 inpatients received psychiatric treatment at our eating-disorder unit, 181 of whom met the DSM-IV-TR criteria for AN during at least one of their stays. One hundred seven $(59.1 \%)$ of the patients with AN had complete data and had given written informed consent to the analysis of their routinely collected data. Thus, the final sample included in this study consisted of 98 female and nine male patients. Illness history was assessed before admission to inpatient treatment, including minimal and maximal lifetime body mass index $\left(\mathrm{BMI}, \mathrm{kg} / \mathrm{m}^{2}\right)$ and age at illness onset. Selfreported weights were verified using medical records. Weightgain during treatment was measured at admission and at discharge as part of the regular treatment protocol. For patients with multiple stays during the study period, the cumulative duration of treatment, the BMI at first admission, and the BMI at last discharge were used. The reported age for all patients is the age at first admission and illness duration represents the time between illness onset and age at first admission. A subsample of 63 patients (female $=61$, male $=2$ ) participated in a followup. For the follow-up measurement, patients who had been discharged for at least 1 year were contacted by e-mail and telephone and asked to complete an online survey. As part of the survey, patients were asked to report their current weight and whether they had sought further treatment after discharge.

\section{Inpatient Treatment}

All study participants were treated at our specialized eatingdisorder unit. The inpatient treatment consists of a multimodal therapy programme with a target $\mathrm{BMI} \geq 18.5 \mathrm{~kg} / \mathrm{m}^{2}$, comprising individual and group psychotherapy, somatic controls and treatment, and structured nutrition increase, with the main goal of normalizing and stabilizing eating behavior and weight. Other therapeutic elements include body-perception therapy, art therapy, nutritional counseling, physiotherapy, and for patients who are advanced in the programme, vocational or educational training and cooking groups. Prior to admission, the indication for hospitalization and illness history is assessed in an detailed medical history interview. Minimal motivation and cooperation for voluntary therapy should be given as the admission to the unit is elective. All patients receive three main meals and three snacks per day with a fixed energy content ranging from 1,600 to $3,000 \mathrm{kcal} /$ day depending on the treatment phase. Patients are required to participate in all elements of the treatment and to gain an average of $700 \mathrm{~g} /$ week until they reach the target BMI. Patients who are unable to adhere to the programme for several weeks have to discontinue therapy. However, as the overarching goal is to rehabilitate the patients as much as possible in their everyday lives, patients may complete treatment in several segments, taking breaks and resuming inpatient treatment at a later time. Between discharge and follow-up, the vast majority of patients $(89 \%)$ received outpatient treatment in form of individual psychotherapy.

\section{Data Analysis}

For the calculation of maximal and minimal lifetime BMI (maximal and minimal BMI hereafter), patients' height at admission and the recalled minimal and maximal lifetime weight after reaching current height were used. Maximal weight suppression was calculated as the difference between maximal $\mathrm{BMI}$ and BMI at admission.

For demographic and clinical data, mean, standard deviation (SD), and range are reported. Percentages are rounded to integers. To compare demographic and weight characteristics between female and male patients, Fisher's exact tests were 
used for the categorical characteristics and Wilcoxon rank sum tests were used to compare continuous characteristics. Bivariate Pearson correlations were calculated to examine the associations among BMI measures (results can be found in the Supplementary Material). To assess the predictive relevance of minimal BMI, maximal BMI, and maximal weight suppression for BMI at admission, at discharge, and at follow-up we fitted linear regression models, estimated using ordinary least squares. First we estimated a base model with the following prognostic parameters as independent variables: age at admission, duration of illness, sex, and BMI at admission (for the prediction of BMI at discharge) and BMI at discharge (for the prediction of BMI at follow-up). Next, minimal or maximal BMI or maximal weight suppression were added as predictors to the basic model to determine the additional variance they explained. To ensure robust estimations of regression coefficients, minimal and maximal BMI or maximal weight suppression were not entered in the same model due to collinearity. Analyses were conducted using $\mathrm{R}$ version 4.0.3 (17). All $p$-values are two-sided and were considered statistically significant at the $5 \%$ level.

\section{RESULTS}

\section{Sample Characteristics}

Demographic and weight characteristics are summarized in Table 1. The female and male patients reported similar minimal and maximal BMI, maximal weight suppression, and a similar proportion of anorexia subtypes, with roughly $1 / 3$ binge-purge and $2 / 3$ restrictive. Female patients showed a slightly higher prevalence of depression compared to male patients (Table 1). During the study period, 38 patients (36\%) were hospitalized more than once (up to five times).

\section{BMI at Admission}

\section{Relationship Between Minimal/Maximal BMI, Weight Suppression and BMI at Admission}

We performed a multiple regression analysis in which the dependent variable was BMI at admission while the independent variables were age, sex, and duration of illness (base model). The model explained a weak proportion of variance $\left(\right.$ adj. $\mathrm{R}^{2}=0.06$ ). Adding minimal BMI to the base model significantly improved the prediction $\left[\mathrm{F}_{(1,102)}=60.95, p<0.0001\right]$, explaining a substantial proportion of variance (adj. $\mathrm{R}^{2}=0.41$ ). Within this model the effect of minimal BMI was significantly positive (Table 2). Adding maximal BMI to the base model did not improve the prediction $\left[\mathrm{F}_{(1,102)}=0.34, p=0.562\right]$. Adding weight suppression to the base model significantly improved the prediction $\left[\mathrm{F}_{(1,102)}=20.87, p<0.0001\right]$, explaining a moderate proportion of variance (adj. $\mathrm{R}^{2}=0.21$ ). Within this model the effect of weight suppression was significantly negative (Table 2).

\section{BMI at Discharge}

\section{Treatment Outcome}

At discharge, $33 \%$ of patients had reached normal weight with a BMI $\geq 18.5 \mathrm{~kg} / \mathrm{m}^{2}$ (good treatment outcome), while $67 \%$ percent were still underweight (intermediate treatment outcome), including 23\% which were severely underweight (BMI
$<16.0 \mathrm{~kg} / \mathrm{m}^{2}$, poor treatment outcome). The proportion of underweight patients was similar between female and male patients (all $p>0.80$ ). Interestingly, the subgroup with severe underweight at discharge showed a history of severe underweight in minimal BMI (Figure 1A), whereas a less clear picture emerged for maximal BMI (Figure 1B).

\section{Relationship Between Minimal/Maximal BMI, Weight Suppression and BMI at Discharge}

We performed a multiple regression analysis in which the dependent variable was BMI at discharge while the independent variables were age, sex, duration of illness, and BMI at admission (base model). The model explained a moderate proportion of variance (adj. $\mathrm{R}^{2}=0.21$ ). Within this model the effect of BMI at admission was significantly positive (Table 2). Adding minimal $\mathrm{BMI}$ to the model did not improve the prediction $\left[\mathrm{F}_{(1,101)}=\right.$ 1.451, $p=0.231$ ]. Adding maximal BMI to the base model significantly improved the prediction $\left[\mathrm{F}_{(1,101)}=5.412, p=\right.$ 0.022 ], explaining a substantial proportion of variance (adj. $\mathrm{R}^{2}$ $=0.24$ ). Within this model the effect of BMI at admission (beta $=0.52,95 \%$ CI $\left.[0.33,0.71], \mathrm{t}_{(101)}=5.39, p<0.001\right)$ and the effect of maximal BMI were significantly positive (Table 2). Adding weight suppression at admission to the base model did improve the prediction $\left[\mathrm{F}_{(1,101)}=5.41, p=0.022\right]$. The model explained a significant and substantial proportion of variance (adj. $\mathrm{R}^{2}=0.24$ ). Within this model, effect of weight suppression at admission was significantly positive (Table 2 ).

\section{BMI at Follow-Up}

\section{Follow-Up Outcome}

Within the subsample of 63 patients who participated in the follow-up, the women reported a lower average BMI at follow-up $($ mean $=17.85(2.12),[12.05,22.86])$ compared to the men (mean $=21.48$ (2.57), [19.67, 23.30], $p=0.043)$. Follow-up took place after an average of 2.89 years $(S D=1.45$, range $=[1.00,5.90])$. At follow-up, $42 \%$ of patients reported a BMI $\geq 18.5 \mathrm{~kg} / \mathrm{m}^{2}$. Of the subsample, $19 \%$ had maintained a $B M I \geq 18.5 \mathrm{~kg} / \mathrm{m}^{2}, 23 \%$ had reached a $B M I \geq 18.5 \mathrm{~kg} / \mathrm{m}^{2}$ after discharge, while another $23 \%$ had lost weight and returned to underweight $\left(\mathrm{BMI}<18.5 \mathrm{~kg} / \mathrm{m}^{2}\right)$, and $34 \%$ were underweight at discharge as well as follow-up. Separating the patients into normal weight (BMI $\geq 18.5 \mathrm{~kg} / \mathrm{m}^{2}$ ), underweight (BMI $<18.5 \mathrm{~kg} / \mathrm{m}^{2}$ ), and severe underweight BMI $\left(<16.0 \mathrm{~kg} / \mathrm{m}^{2}\right)$ by their BMI at follow-up, there was no evidence for differences between these subgroups of BMI at admission or discharge (Figure 2).

\section{Relationship Between Minimal/Maximal BMI, Weight Suppression and BMI at Follow-Up}

Finally, we performed a multiple regression analysis in which the dependent variable was BMI at follow-up while the independent variables were age, sex, duration of illness, BMI at admission, and BMI at discharge (base model). The model explained a non-significant and weak proportion of variance $\left(\operatorname{adj} . \mathrm{R}^{2}=\right.$ -0.007). Adding minimal BMI to the model did not improve the prediction $\left[\mathrm{F}_{(1,57)}=3.00, p=0.089\right]$. Adding maximal BMI to the base model significantly improved the prediction $\left[\mathrm{F}_{(1,57)}=5.09, p=0.028\right]$, however the model explained only 
TABLE 1 | Demographic and weight characteristics.

\begin{tabular}{|c|c|c|c|}
\hline \multirow[b]{2}{*}{ Variable } & \multicolumn{2}{|c|}{ Sex } & \multirow[b]{2}{*}{$p$-value } \\
\hline & $\begin{array}{c}\text { Male } \\
n=9 \\
\text { Mean (SD) [Range]/n(\%) }\end{array}$ & $\begin{array}{c}\text { Female } \\
n=98 \\
\text { Mean (SD) [Range]/n (\%) }\end{array}$ & \\
\hline Age (years) & $24.14(5.58)[17.19,34.52]$ & $24.86(8.44)[17.00,55.77]$ & 0.9 \\
\hline Age at illness onset (years) & $18.44(3.88)[14.00,24.00]$ & $17.17(5.84)[10.00,46.00]$ & 0.2 \\
\hline Illness duration (years) & $5.70(5.33)[1.15,17.52]$ & $7.71(7.32)[0.50,41.77]$ & 0.5 \\
\hline BMl at admission & 15.96 (1.33) [13.40, 18.20] & 14.55 (1.65) [10.60, 18.30] & 0.023 \\
\hline $\mathrm{BMl}$ at discharge & $17.80(1.61)[15.80,20.10]$ & $17.32(1.84)[11.90,20.40]$ & 0.6 \\
\hline Min. BMl & $14.19(1.50)[11.00,16.00]$ & $13.52(1.67)[10.00,17.50]$ & 0.15 \\
\hline Max. BMl & $21.61(3.49)[17.00,28.00]$ & $20.76(3.43)[15.60,39.00]$ & 0.4 \\
\hline Weight suppression & 5.66 (3.03) [1.70, 11.90] & $6.21(3.67)[0.20,22.40]$ & 0.6 \\
\hline AN type & & & $>0.9$ \\
\hline binge-purge & $3(33 \%)$ & $34(35 \%)$ & \\
\hline restrictive & $6(67 \%)$ & $64(65 \%)$ & \\
\hline Comorbid depression & $2(22 \%)$ & 58 (59\%) & 0.041 \\
\hline
\end{tabular}

${ }^{a}$ Wilcoxon rank sum test; Fisher's exact test. In bold, $p$-values $<0.05$.

TABLE 2 | Summary of regression models for BMl at admission, discharge and follow-up.

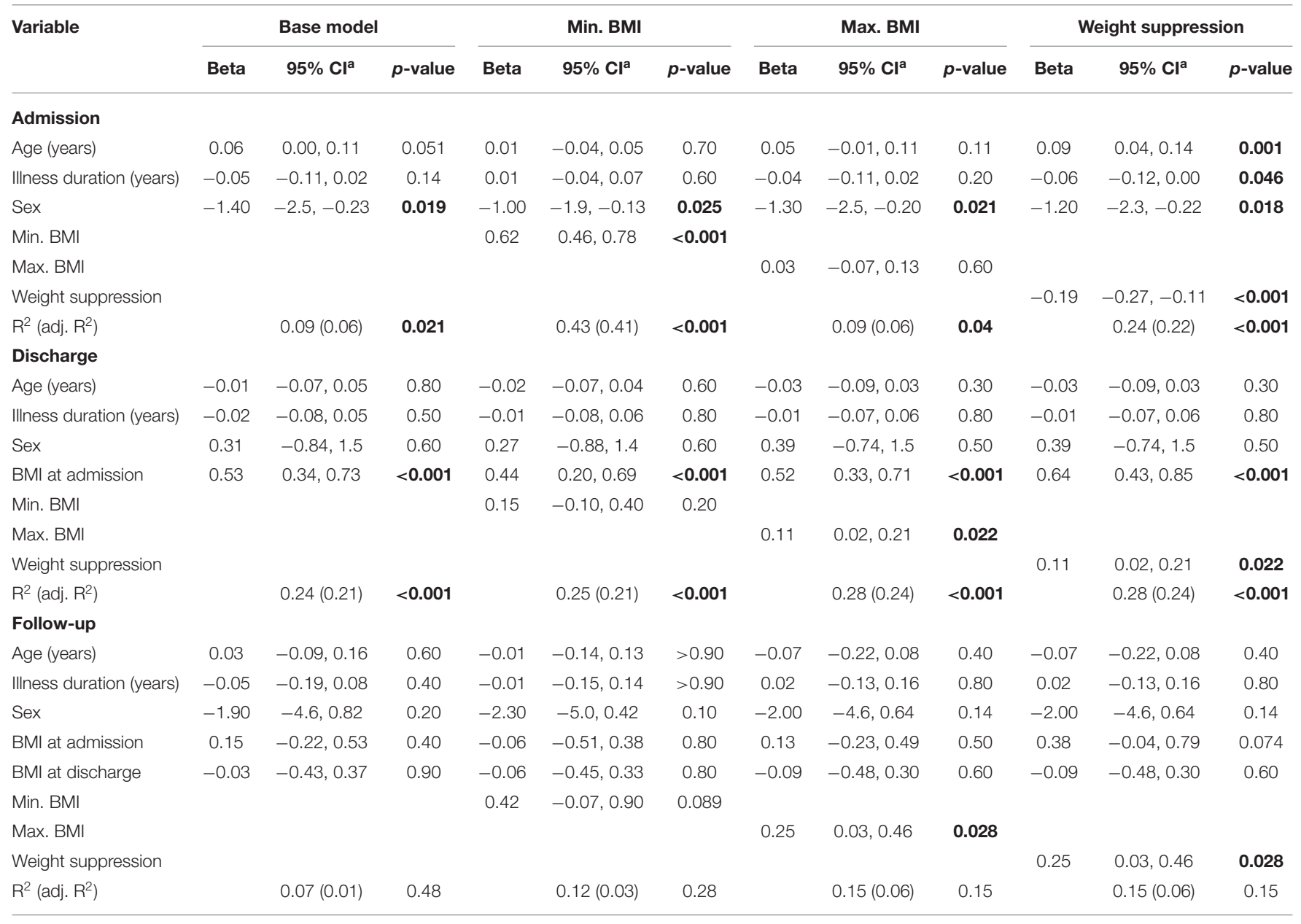

${ }^{a} \mathrm{Cl}$, Confidence Interval. In bold, $p$-values $<0.05$. 

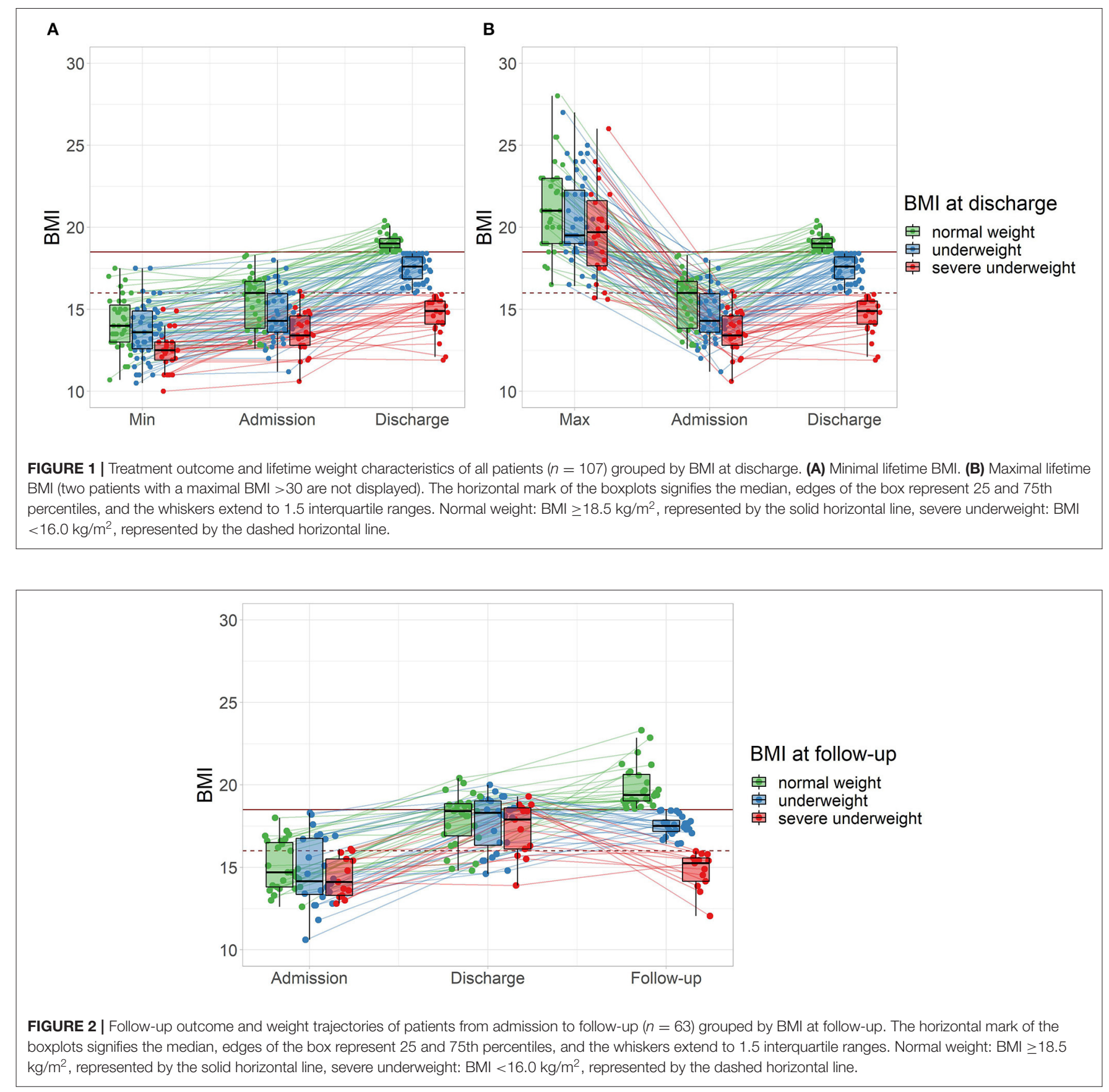

a non-significant proportion of variance (adj. $\mathrm{R}^{2}=0.06$ ). Within this model the effect of maximal BMI was significantly positive (Table 2). Adding weight suppression at admission to the base model did improve the prediction $\left[\mathrm{F}_{(1,57)}=5.09, p=0.028\right]$. The model explained a not significant and moderate proportion of variance (adj. $\mathrm{R}^{2}=0.06$ ). Within this model, effect of weight suppression at admission was significantly positive (Table 2 ). Similar multiple regression results were seen for all follow-up models when including time between discharge and follow-up (time to follow-up) as covariate. Time to follow-up did not significantly alter the model predictions (all $F<1.44, p>0.24$ ) and had no significant effect on BMI at follow-up (all $t<$ $1.20, p>0.24)$.

\section{DISCUSSION}

The BMI is a critical marker of illness severity in AN and is widely considered a key predictor of treatment outcome in adolescent, however detailed analysis of the predictive value of BMI history in adult patients has been lacking. In the present study, we examined the lowest and highest lifetime BMI, and the weight suppression at admission as predisposing factors for the outcome of inpatient 
treatment in adult patients with AN. Specifically, we analyzed the relationship of minimal $\mathrm{BMI}$, maximal $\mathrm{BMI}$, and maximal weight suppression with the BMI at admission, discharge, and follow-up, while controlling for other parameters of illness history.

Our results showed a strong association of minimal lifetime BMI and BMI at admission, even when considering patients' age, sex, and duration of illness. An increment of $1.0 \mathrm{~kg} / \mathrm{m}^{2}$ in minimal BMI was associated with a mean increase of $0.62 \mathrm{~kg} / \mathrm{m}^{2}$ in BMI at admission. Higher weight suppression contributed moderately to the prediction of lower BMI at admission when controlling for age, sex, and duration of illness, whereas maximal BMI had no predictive power for the BMI at admission. This indicates, similar to the premorbid BMI in adolescents $(6,18)$, that minimal lifetime BMI is a strong predictor for the weight status at admission in adults. For the BMI at discharge, BMI at admission and the parameters of illness history together explained $21 \%$ of the variance, with BMI at admission being the strongest outcome predictor. Minimal BMI added little information to this. However, maximal BMI and weight suppression improved this prediction independently of BMI at admission, with a $1.0 \mathrm{~kg} / \mathrm{m}^{2}$ increase in maximal BMI or weight suppression being associated with a $0.11 \mathrm{~kg} / \mathrm{m}^{2}$ increase in BMI at discharge. The counterintuitive association of higher weight suppression as beneficial predictor is consistent with previous reports of a positive association of weight suppression and weight gain during inpatient $(12,13,19)$ and outpatient treatment (20). Given the high correlation between weight suppression and maximal BMI, it stands to reason that the beneficial effect of weight suppression is driven by maximal BMI.

Finally, the BMI at follow-up was not predictable by BMI at admission or BMI at discharge. Minimal BMI was significantly correlated with BMI at follow-up, but added no additional information when controlling for the other variables. However, higher maximal BMI or weight suppression of $1.0 \mathrm{~kg} / \mathrm{m}^{2}$ was associated with a $0.25 \mathrm{~kg} / \mathrm{m}^{2}$ increase in BMI at follow-up. The lack of predictive power of the BMI at discharge is in contrast to reports of a 6-month follow-up (21), however this difference might be explained by the longer time to follow-up in our study. Consistent with our results, the above-mentioned study reports low predictive power for the minimal BMI (21). Maximal BMI itself has not been considered as predictor of follow-up BMI in previous research, but appears to be the driving force behind weight suppression at admission given their high correlation. The positive predictive power of weight suppression is in line with reports on adolescents with AN (14), where greater weight suppression at lowest BMI predicted higher BMI at 6-, 10-, and 18-year follow-up.

Taken together, a low minimal lifetime BMI seems to have a negative influence on the weight at admission, which in turn results in a lower BMI at discharge. Higher maximal BMI had a positive influence on BMI at discharge, and at follow-up maximal BMI had become more important than BMI at admission or discharge, contributing significantly to a higher weight. Overall, maximal BMI emerged as the most important factor in predicting the course of AN. While the underlying mechanism for this is unclear, lower maximal BMI may reflect metabolic aspects of the illness, such as a genetic predisposition to lower body fat, which is known to contribute to the etiology of AN $(22,23)$. From a clinical point of view, our therapeutic experience suggests that a maximal lifetime BMI within a normal range can positively influence the course of weight gain treatment. It is conceivable that for patients who have had body experiences with weight in the normal range, therapeutic weight gain up to a know weight is more imaginable and thus easier to achieve.

In recent years, the concept of the weight suppression, as the difference between maximal BMI and current or lowest BMI, has gained attention. Our results support the notion that greater weight suppression at admission is associated with higher BMI at discharge and better weight maintenance at follow-up. Considering the constituents of weight suppression that may drive its predictive power (10), it is apparent that maximal lifetime BMI is the key factor in the present study. Therefore, given the law of parsimony (Occam's razor), it seems most important to determine the maximal BMI in order to predict treatment outcome and BMI at follow-up in patients with AN.

The longer duration of illness in our adult sample did not emerge as meaningful predictors of treatment outcome or outcome at follow-up. While duration of illness is a known influence on long-term trajectories of $\mathrm{AN}$ [e.g., $(3,24)]$, this is in line with follow-up reports assessing treatment outcome at 6month (21) and 1-year follow-up (6). Of concern, although in line with the literature (25-27), is that more that the half of the patients had remained underweight or returned to underweight at follow-up. These troubling findings underline the need of new therapeutic strategies to better treat severely ill patients and prevent relapse, and intensify research in this field (28).

One of the limitations of the present study is that a direct comparison between minimal BMI, maximal BMI and premorbid BMI is only partially possible. However, in adult samples, it is often not possible to determine the premorbid BMI since information on height at illness onset are not available. Instead, the minimal and maximal lifetime BMI are readily available data that are well-remembered by patients. Minimal and maximal lifetime BMI were assessed using self-reported values from the medical history interview. While all information was carefully checked against the medical records, biased reporting cannot fully be ruled out. However, as weight is inherently a key information to anorexia nervosa, patients are very accurate in reporting their weights $(29,30)$. The present study focussed on BMI as measure of treatment and follow-up outcome. While $\mathrm{BMI}$ is a core outcome measure in $\mathrm{AN}$, the authors note that psychiatric and psychological aspects also play an important role and should be considered in future studies.

To conclude, our results suggest that a lower minimal lifetime BMI presents a negative prognostic factor in the short-term, promoting a lower BMI at admission. In contrast, a higher maximal lifetime BMI proved to be a positive prognostic factor in the medium and long-term, promoting better treatment outcomes even years after discharge. In addition, the very high correlation between maximal BMI and weight suppression at admission emphasizes the role of maximal BMI in weight trajectories. These findings highlight the importance of considering both the lower and especially the upper end 
of the lifetime weight range when treating adult patients with severe AN.

\section{DATA AVAILABILITY STATEMENT}

The raw data supporting the conclusions of this article will be made available by the authors, without undue reservation.

\section{ETHICS STATEMENT}

The studies involving human participants were reviewed and approved by Kantonale Ethikkommission Zürich. The patients/participants provided their written informed consent to participate in this study.

\section{AUTHOR CONTRIBUTIONS}

L-KK: conceptualization, data curation, formal analysis, visualization, writing-original draft, and review \& editing. HM: formal analysis and writing-review \& editing. GM:

\section{REFERENCES}

1. Watson HJ, Bulik CM. Update on the treatment of anorexia nervosa: review of clinical trials, practice guidelines and emerging interventions. Psychol Med. (2013) 43:2477-500. doi: 10.1017/S0033291712002620

2. NICE. Eating Disorders. Core Interventions in the Treatment and Management of Anorexia Nervosa, Bulimia Nervosa and Related Eating Disorders. Leicester, London: The British Psychological Society \& The Royal College of Psychiatrists (2004).

3. Steinhausen H-C. The outcome of anorexia nervosa in the 20th Century. Am J Psychiatry. (2002) 159:1284-93. doi: 10.1176/appi.ajp.159.8.1284

4. Stice E, Gau JM, Rohde P, Shaw H. Risk factors that predict future onset of each DSM-5 eating disorder: predictive specificity in high-risk adolescent females. J Abnorm Psychol. (2017) 126:38-51. doi: 10.1037/abn0 000219

5. Yilmaz Z, Gottfredson NC, Zerwas SC, Bulik CM, Micali N. Developmental premorbid body mass index trajectories of adolescents with eating disorders in a longitudinal population cohort. J Am Acad Child Adolesc Psychiatry. (2019) 58:191-9. doi: 10.1016/j.jaac.2018.11.008

6. Föcker M, Bühren K, Timmesfeld N, Dempfle A, Knoll S, Schwarte R, et al. The relationship between premorbid body weight and weight at referral, at discharge and at 1-year follow-up in anorexia nervosa. Eur Child Adolesc Psychiatry. (2015) 24:537-44. doi: 10.1007/s00787-014-0605-0

7. Miyasaka N, Yoshiuchi K, Yamanaka G, Sasaki T, Kumano H, Kuboki T. Relations among premorbid weight, referral weight, and psychological test scores for patients with anorexia nervosa. Psychol Rep. (2003) 92:6774. doi: 10.2466/pr0.2003.92.1.67

8. Steinhausen H-C, Grigoroiu-Serbanescu M, Boyadjieva S, Neumärker K-J, Metzke CW. The relevance of body weight in the medium-term to long-term course of adolescent anorexia nervosa. Findings from a multisite study. Int $J$ Eat Disord. (2009) 42:19-25. doi: 10.1002/eat.20577

9. Hebebrand J. Identification of determinants of referral and follow-up body mass index of adolescent patients with anorexia nervosa: evidence for the role of premorbid body weight. Eur Child Adolesc Psychiatry. (2015) 24:4715. doi: 10.1007/s00787-015-0711-7

10. Lowe MR, Piers AD, Benson L. Weight suppression in eating disorders: a research and conceptual update. Curr Psychiatry Rep. (2018) 20:80. doi: 10.1007/s11920-018-0955-2

11. Stice E, Rohde P, Shaw H, Desjardins C. Weight suppression increases odds for future onset of anorexia nervosa, bulimia nervosa, and purging conceptualization, writing-review \& editing, and funding acquisition. All authors contributed to the article and approved the submitted version.

\section{ACKNOWLEDGMENTS}

The authors would like to thank all patients for their contribution to this study and all members of the research and therapy team for their support, in particular Valeria Vincenti and Meret Wittlin for their assistance with data collection and Elena Margiotta and Ramona Kühne for their support with data quality control. Further, we gratefully acknowledge the financial support of this work from the Palatin Foundation and the USZ Foundation.

\section{SUPPLEMENTARY MATERIAL}

The Supplementary Material for this article can be found online at: https://www.frontiersin.org/articles/10.3389/fpsyt. 2021.682952/full\#supplementary-material

disorder, but not binge eating disorder. Am J Clin Nutr. (2020) 112:9417. doi: $10.1093 /$ ajcn/nqaa146

12. Wildes JE, Marcus MD. Weight suppression as a predictor of weight gain and response to intensive behavioral treatment in patients with anorexia nervosa. Behav Res Ther. (2012) 50:266-74. doi: 10.1016/j.brat.2012.02.006

13. Piers AD, Espel-Huynh HM, Lowe MR. The independent and interacting effects of weight suppression and admission body mass index on treatment weight change in patients with anorexia nervosa or bulimia nervosa. Int J Eat Disord. (2019) 52:1301-9. doi: 10.1002/eat.23149

14. Witt AA, Berkowitz SA, Gillberg C, Lowe MR, Råstam M, Wentz E. Weight suppression and body mass index interact to predict long-term weight outcomes in adolescent-onset anorexia nervosa. J Consult Clin Psychol. (2014) 82:1207-11. doi: 10.1037/a0037484

15. Bodell LP, Racine SE, Wildes JE. Examining weight suppression as a predictor of eating disorder symptom trajectories in anorexia nervosa. Int $J$ Eat Disord. (2016) 49:753-63. doi: 10.1002/eat.22545

16. Uniacke B, Attia E, Kaplan A, Walsh BT. Weight suppression and weight maintenance following treatment of anorexia nervosa. Int J Eat Disord. (2020) 53:1002-6. doi: 10.1002/eat.23269

17. R Core Team. R: A Language and Environment for Statistical Computing. Vienna, Austria: R Foundation for Statistical Computing (2021).

18. Coners H, Remschmidt H, Hebebrand J. The relationship between premorbid body weight, weight loss, and weight at referral in adolescent patients with anorexia nervosa. Int J Eat Disord. (1999) 26:171-8. doi: 10.1002/(sici)1098108x(199909)26:2<171::aid-eat6>3.0.co;2-pc

19. Berner LA, Shaw JA, Witt AA, Lowe MR. The relation of weight suppression and body mass index to symptomatology and treatment response in anorexia nervosa. J Abnorm Psychol. (2013) 122:694-708. doi: 10.1037/a0033930

20. Carter FA, Boden JM, Jordan J, McIntosh VVW, Bulik CM, Joyce PR. Weight suppression predicts total weight gain and rate of weight gain in outpatients with anorexia nervosa. Int J Eat Disord. (2015) 48:9128. doi: 10.1002/eat.22425

21. Redgrave GW, Schreyer CC, Coughlin JW, Fischer LK, Pletch A, Guarda AS. Discharge body mass index, not illness chronicity, predicts 6-month weight outcome in patients hospitalized with anorexia nervosa. Front Psychiatry. (2021) 12:641861. doi: 10.3389/fpsyt.2021.641861

22. Watson HJ, Yilmaz Z, Thornton LM, Hübel C, Coleman JRI, Gaspar HA, et al. Genome-wide association study identifies eight risk loci and implicates metabo-psychiatric origins for anorexia nervosa. Nat Genet. (2019) 51:120714. doi: 10.1038/s41588-019-0439-2 
23. Duncan L, Yilmaz Z, Gaspar H, Walters R, Goldstein J, Anttila V, et al. Significant locus and metabolic genetic correlations revealed in genomewide association study of anorexia nervosa. Am J Psychiatry. (2017) 174:8508. doi: 10.1176/appi.ajp.2017.16121402

24. Fichter MM, Quadflieg N, Crosby RD, Koch S. Long-term outcome of anorexia nervosa: results from a large clinical longitudinal study. Int J Eat Disord. (2017) 50: 1018-30. doi: 10.1002/eat.22736

25. Zipfel S, Giel KE, Bulik CM, Hay P, Schmidt U. Anorexia nervosa: aetiology, assessment, and treatment. Lancet Psychiatry. (2015) 2:1099111. doi: 10.1016/S2215-0366(15)00356-9

26. Kaye W. Eating disorders: hope despite mortal risk. Am J Psychiatry. (2009) 166:1309-11. doi: 10.1176/appi.ajp.2009.09101424

27. Treasure J, Claudino AM, Zucker N. Eating disorders. Lancet. (2010) 375:58393. doi: 10.1016/S0140-6736(20)30059-3

28. Treasure J, Oyeleye O, Bonin E, Zipfel S, Fernandez-Aranda F. Optimising care pathways for adult anorexia nervosa. What is the evidence to guide the provision of high-quality, cost-effective services? Eur Eat Disord Rev. (2021) 29:306-15. doi: 10.1002/erv.2821
29. McCabe RE, McFarlane T, Polivy J, Olmsted MP. Eating disorders, dieting, and the accuracy of self-reported weight. Int J Eat Disord. (2001) 29:59-64. doi: 10. 1002/1098-108x(200101)29:1<59::aid-eat9>3.0.co;2-\#

30. Meyer C, Arcelus J, Wright S. Accuracy of self-reported weight and height among women with eating disorders: a replication and extension study. Eur Eat Disord Rev. (2009) 17:366-70. doi: 10.1002/erv.950

Conflict of Interest: The authors declare that the research was conducted in the absence of any commercial or financial relationships that could be construed as a potential conflict of interest.

Copyright $\odot 2021$ Kaufmann, Moergeli and Milos. This is an open-access article distributed under the terms of the Creative Commons Attribution License (CC BY). The use, distribution or reproduction in other forums is permitted, provided the original author(s) and the copyright owner(s) are credited and that the original publication in this journal is cited, in accordance with accepted academic practice. No use, distribution or reproduction is permitted which does not comply with these terms. 